\title{
LESS FREQUENT NEUROLOGIC COMPLICATIONS OF ANTICOAGULANT THERAPY
}

\author{
Radomír Taláb, Gerhard Waberžinek \\ Charles University in Prague, Faculty of Medicine in Hradec Králové: Department of Neurology \\ Summary: One of the less frequent complications of anticoagulant therapy is damage to the femoral and sciatic nerves due \\ to bleeding. This report presents two cases and discusses the pathogenetic mechanism and treatment of this medical pro- \\ blem.
}

Key words: Anticoagulant therapy; Femoral nerve; Sciatic nerve

Anticoagulant therapy may cause complications despite its positive therapeutic effects. The most important neurologic complications are subarachnoid hemorrhage, subdural hematoma, intracerebral or cerebellar hemorrhage, spinal epidural hematoma, and lesions of certain peripheral nerves usually due to compression caused by intramuscular hematoma. Among the less frequent complications are certain lesions of the femoral and sciatic nerves $(2,4,7-8)$. The following 2 cases indicate the necessity to take also these complications into our differential diagnostic consideration.

\section{Patient 1.}

A 41 year-old woman had been repeatedly treated during her pregnancy for deep venous thrombosis in her left lower extremity. Thirteen years later, during another pregnancy, she again developed deep phlebothrombosis in the same leg. After treatment with heparin (first 30,000 units $/ 24 \mathrm{~h}$, then 60,000 units $/ 24 \mathrm{~h}$ ), thrombolysis was performed with a total dose of 3.5 mil. units of Streptase. Five hours following thrombolysis the patient aborted the dead foetus. Due to massive uterine hemorrhage instrumental exploration of the uterus was performed, and anticoagulant therapy with heparin and warfarin was continued. Thrombophlebitic changes on the left leg had improved, but the course was complicated by about of endomyometritis which was treated with antibiotics. Ten days later pain in the right lower extremity had developed with paresthesias along the medial aspect of the thigh down to the ankle, with impairment of motility. A fresh complete denervation of the right rectus and lateral vastus muscles was diagnosed clinically, and on electromyography (EMG) the occurrence of multiple denervations with no response to stimulation was found. A control ultrasound examination of the abdo- men and retroperitoneum 14 days after the beginning of pain was unremarkable.

A CT scan of the pelvis one month later revealed in two projections at $8 \mathrm{~mm}$ a somewhat hyperdense focus in the area of the right inguinal band at the dorsolateral margin of the psoas muscle; this density could have corresponded to a residue of an absorbing hematoma. Conservative treatment was provided.

The clinical condition of the patient gradually improved, pain in the lower extremity had subsided, but a residual lesion of the femoral nerve remained. Systematic rehabilitation was instituted; after 6 months the patient was able to walk unassisted with the use of a short can due to residual paralysis of her right leg. An EMG examination 8 $1 / 2$ months later revealed a still (severely) reduced contraction curve at rest with the occurrence of wide polyphasic action potentials. At present the patient is able to walk unassisted, without support (Fig. 1).

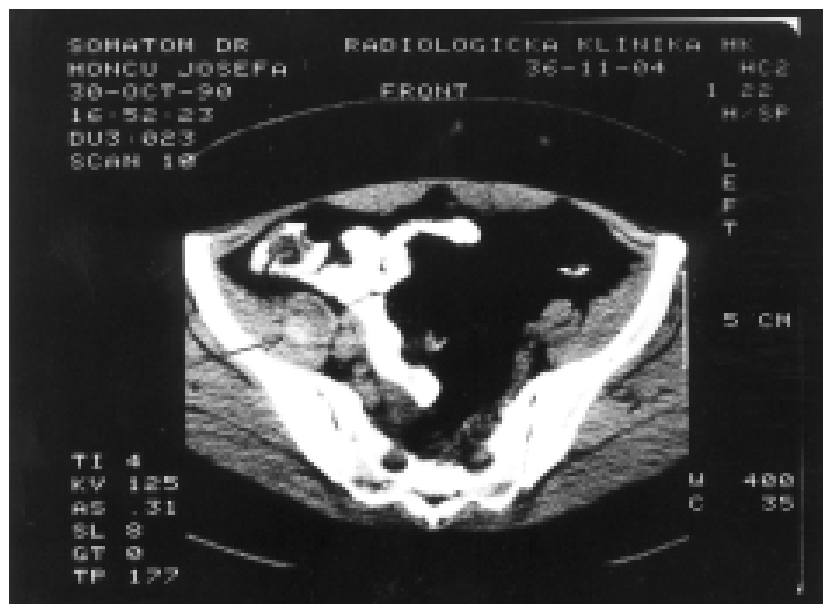

Fig. 1 


\section{Patient 2.}

A 21 year-old woman was found unconscious due to severe drug intoxication from an attempt to commit suicide. She was brought to the hospital on April 5, 1990, where hemoperfusion and hemodialysis were performed, following which the patient had regained her consciousness. On the following day a hematoma was found in her right gluteal region; a day later diffuse pain had developed in her right lower extremity with hypesthesia of the toes and inability to dorsiflect her right foot. This finding indicated a lesion of the right sciatic nerve most probably due to pressure from an intrapelvic hematoma. A diagnostic tap of this area yielded negative results.

During the following days signs of a fully developed peroneo-tibial paralysis became manifest, severe pain of the right lower extremity persisted, arterial pulsations dimnished acrally. A repeat ultrasound examination of the gluteal region and retroperitoneum was normal. A CT scan of the pelvis and the proximal right thigh revealed soft tissue contusion in the area of the right buttock and thigh with diffuse hemorrhage.

Presacrally an older, organized hematoma was depicted (Fig. 2). Conservative treatment was offered. The pain and hypesthesias subsided gradually with a persisting severe residual peroneo-tibial paresis of the right leg. The patient was able to walk only with the aid of two French canes; five months following the sciatic nerve lesion the patient, a nurse, was able to resume her profession; she was able to walk without assistance, but with persisting pain in her right leg. She has developed a passive block in her right ankle joint with a shortening of the Achilles tendon. Reexamination after one year revealed considerable improvement in her clinical condition; on electromyography degenerative potentials were found in all examined muscles, particularly of the peroneal group. In the neighboring muscles contraction curve was reduced; in the right gastrocnemius muscle reinnervational action potentials were observed. Eventually the patient fully recovered (Fig. 2).

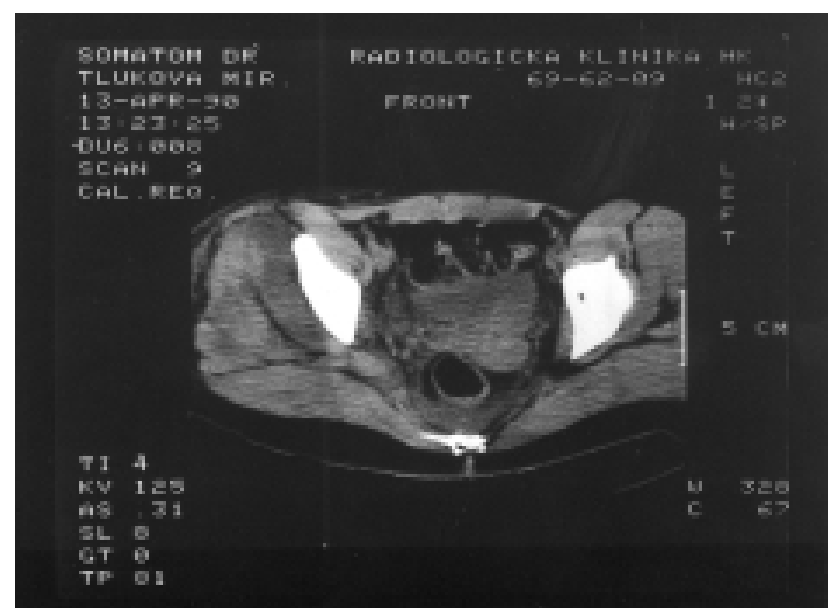

Fig. 2

\section{Discussion}

Damage to the lumbosacral plexus, and particularly to its main nerve trunks - the femoral and sciatic nerves - cannot be regarded unusual, if one also considers certain radicular lesions accompanying lumbar discopathies. Less frequent are certain pathogenic mechanisms with selective damage to the femoral and sciatic nerves; but the topographic anatomic relations of these structures predispose them to potential damage.

The predisposing location for damage to the femoral nerve is the area extending from its entry into the fissure between the iliac and psoas muscles to the exit of the nerve from the pelvis under the inguinal ligament through the lacuna musculorum (4) (Fig. 3).

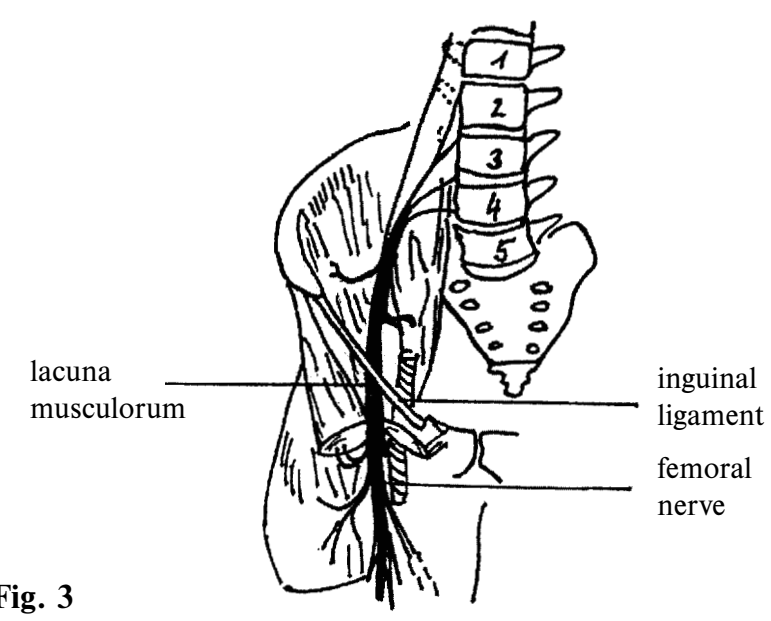

Nerve fibers of the sciatic nerve converge in the sciatic foramen (foramen ischiadicum maius), about the piriform muscle, and exit the pelvis through the infrapiriform opening as the fully constituted sciatic nerve. If we omit lumbar discopathies and malformations of the lumbosacral spine, the sciatic nerve inside the pelvis predisposes to damage up to its exit through the infrapiriform foramen (4) (Fig. 4).

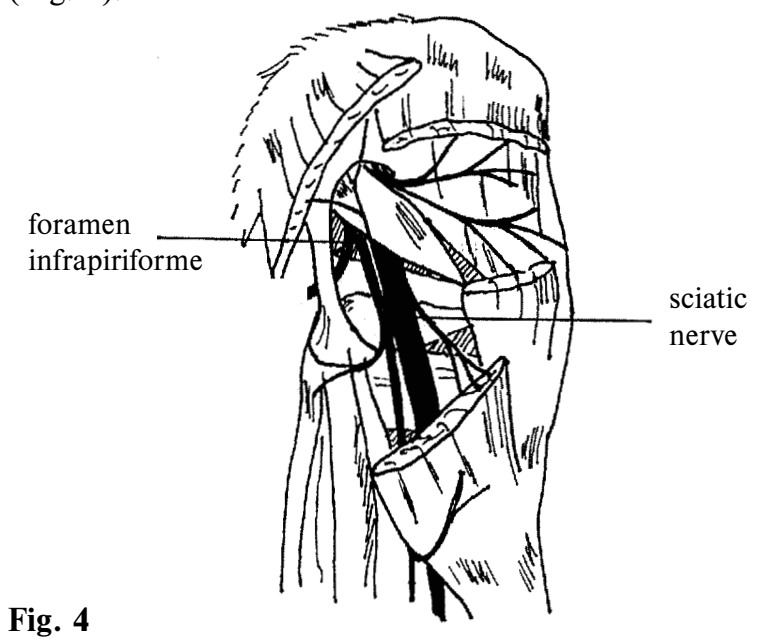


In addition to topography, other important factors - determining the development of nerve lesions and the clinical syndrome - are pathogenic mechanisms summarized in tables 1 and 2.

Tab. 1: Etiopathogenic mechanisms of damage to the femoral nerve.

\begin{tabular}{|ll|}
\hline Traumatic & Non-traumatic \\
\hline $\begin{array}{l}\text { bullet and stab wounds } \\
\text { hyperextension, rupture } \\
\text { fractures }\end{array}$ & tumors \\
& inflammatory processes \\
abscesses & lymphadenopathies \\
aneurysm of the external & iliac artery \\
& dyscrasial mononeuritis \\
& idiopathic mononeuritis \\
& toxic mononeuritis \\
& hematomas \\
& Iatrogenic mechanisms \\
& hematomas \\
surgical procedures & \\
catheterization of the femoral artery & \\
delivery with forceps & \\
\hline
\end{tabular}

Tab. 2: Etiopathogenic damage to the sciatic nerve.

\begin{tabular}{|c|c|}
\hline Traumatic & Non-traumatic \\
\hline $\begin{array}{l}\text { bullet wounds, stabs, cuts, slashes } \\
\text { excessive stretching } \\
\text { fractures } \\
\text { external compression }\end{array}$ & $\begin{array}{l}\text { pregnancy } \\
\text { protracted childbirth } \\
\text { tumors (carcinomas, } \\
\text { metastases) } \\
\text { inflammations, abscesses } \\
\text { lymphadenopathies } \\
\text { echinococcal cyst } \\
\text { aneurysms } \\
\text { (inferior gluteal artery) } \\
\text { hematomas } \\
\text { discopathies, spondylosis } \\
\text { polyneuritis } \\
\text { vasculitis }\end{array}$ \\
\hline \multicolumn{2}{|l|}{$\begin{array}{l}\text { intrathecal injection } \\
\text { intramuscular injection } \\
\text { positional compression }\end{array}$} \\
\hline
\end{tabular}

The diagnosis of direct lesions to the femoral and sciatic nerve is usually not difficult. These injuries involve contusions, open stab wounds, cuts, or bullet wounds. Similar mechanisms are iatrogenic injuries to the neural trunk (femoral nerve) representing complications during surgery, such as appendectomy, lumbar sympathectomy, removal of an intraabdominal lipoma, inguinal hernia repair, femoropopliteal bypass, or forceps delivery. A less traumatic mechanism, such as an intramuscular injection in the gluteal or femoral region, may also lead to lesions of the neural trunk of both nerves. Hence external mechanisms are common for all these types of injuries (Tables 1 and 2).

The underlying cause of the non-traumatic (endogenous) mechanisms of damage to the neural trunks of the femoral and sciatic nerves is ultimately also a consequence of compression or contusion of the nerve trunk. This type of damage occurs in pregnancy, during protracted childbirth, from pressure of a tumor, from bone splinters in fractures of the femur ( sciatic nerve), or from compression by retroperitoneal and pelvic lymphadenopathies, psoatic abscesses, or exudates in appendicitis (femoral nerve).

Less frequent are lesions of these neural trunks from different types of polyneuritides, blood dyscrasias (diabetes, gout), toxic (alcoholism) and allergic neuritis of the femoral nerve. The sciatic nerve may be affected by extension of inflammation from the pelvis (in parametritis, abscess), or even endometriosis of the nerve. Both nerves may be damaged by compression from a hematoma in anatomically predisposing locations; either from spontaneous hemorrhage (coagulopathies), or iatrogenically, most often in connection with anticoagulant therapy - as in our 2 cases.

In hemophilia, hemarthrosis and contractures are common complications; less freequent is intracranial bleeding. Extremely rare are cases of bleeding into the spinal canal and the development of a so called pseudotumor in the spinal canal, in the area of the pelvis and upper thigh (proximal pseudotumor), or on the arms and legs (distal pseudotumor). These pseudotumors, which are often multiple, are seen mostly in children (6). Iatrogenic lesions of the femoral and sciatic nerves are uncommon but wellknown complications of anticoagulant therapy. The reason for bleeding and the formation of hematoma is not entirely clear.

Complicated bleeding usually occurs as a consequence of a bolus or overdose of anticoagulants, or from long-term therapy with anticoagulants. The provoking mechanism for bleeding is believed to be the same as in hemophiliacs in whom intramuscular bleeding into the psoas muscle may occur as a consequence of stretching, jumping, or during manipulation with the spinal column. Fascias of the iliac and psoas muscles are separated except in the region under the inguinal ligament, hence enabling the formation of subfascial hematoma between the two muscles, causing damage to the femoral nerve.

Another possible mechanism may be infiltration of blood directly into the nerve fibers.

In our 2 patients we suspected an initial mechanical (external) component. In the first, the formation of subfascial hemorrhage in the ilieopsoas muscle in connection with instrumental exploration of the uterine cavity following postabortional bleeding. A causal relation between endomyometritis and lesion of the femoral nerve appeared less 
probable. In the second case hemoperfusion and hemodialysis was performed due to drug intoxication; at the same time certain medications were given intramuscularly. Hypothetically we may consider a transfascial penetration of blood from the gluteal region all the way into the infrapiriform foramen with a subsequent lesion of the sciatic nerve. The common denominator in both cases was obviously the application of large doses of anticoagulants.

In the first patient long-term anticoagulation was given despite complicating hemorrhage, in order to prevent the recurrence of deep venous thrombosis. This may explain the late (after 1 month) visualization of what appears like a residue of a subfascial hematoma on CT scan (Fig. 1). In the second patient there was only a one time aplication of large doses of anticoagulants during hemoperfusion and dialysis.

\section{Conclusions}

We wanted to draw attention to one of the less frequent, but well-known complications of anticoagulant therapy - damage to the femoral and sciatic nerves.

In the diagnosis and therapy of these complications early clinical diagnosis - i.e. CT of the region of the lesion with visualization of the hematoma - and its subsequent early evacuation, are essential. This way one may increase the probability of fast and complete recovery of the lost nerve functions and prevent a severe denervation syndrome.

\section{Acknowledgements}

We thank Miroslav Šercl for CT scan, František Pára for EMG, Blanka Hawlikova for secretarial help.

\section{References}

1. Biemond A. Femoral neuropathy. In: Vinken PJ and Bruyn GW. Handbook of Neurology, 8,part II, 1970:303-20

2. Butterfield WC, Nevaiser RJ, Roberts MP. Femoral Neuropathy and Anticoagulants, Ann Surg 1971;176:58-61.

3. Gilroy J, Meyer JS. Medical Neurology,1979:700-14

4. Hrbek J. Neurology 1986;36:277-314.

5. Salazar-Grueso E, Roos R. Sciantic endometriosis: A treatable sensorimotor mononeuropathy. Neurology 1986;36:1360-3.

6. Shih Sing Liu, White WL, Johnson PC, Guantt Ch. Hemophilic pseudotumor of the spinal canal. J Neurosurg 1988;69:624-7.

7. Stewart-Wynne EG. Iatrogenic femoral neuropathy. Br Med J 1976;31:263.

8. Zvěřina E, Stejskal L. Damages of peripheral nerves. Prague: Avicenum, 1979:227-34

Submitted July 1999.

Accepted September 1999.

MUDr. Radomír Taláb, CSc.,

Charles University in Prague, Faculty of Medicine in Hradec Králové,

Department of Neurology, 50005 Hradec Králové,

Czech Republic. e-mail: talab@fnhk.cz 